\title{
I N DEX
}

Note: literary works can be found under author's names. Page numbers in italic refer to illustrations.

abjection; the abject 169-70, 173

see also Kristeva, Julia

adolescence; puberty 12, 155, 163-4, 165-7, $168,169,175,214-15,222$

see also teenagers; Young Adult fiction advertisements see fashion

Aesop 8, 22-3, 32n.3, 39, 40, 41-2, 46 n. 20

see also fables

Afanas'ev, Alexander Nikolayevich see fairy tales

agency; autonomy 4, 6, 7, 12, 17n.25, 45, 130, 150, 151, 153-4, 155, 159, 16on.14, 224

see also subjectivity

Alas, Mert 238-9, 239

alienation $61,149,154,157,167$

see also commodification; reification

Amala and Kamala see wolf-children

An American Werewolf in London (1981 film) 10, 71, 8o-3, 185, 188-9n.9, 230

Andersen, Hans Christian see fairy tales androgyny 232, 234

see also femininity; gender; masculinity; transvestism

animal(s) 1, 7-8, 9, 31, 43, 95-6, 113, 147, 162n.38, 163, 164, 168, 171, 173, 195-201, 203, 213-14, 217-18, 223-4, 232

bat(s) 76,83 n.6, 195-6, 209n.8

bear(s) 3, 38, 49, 50, 51, 56, 65n.5, 90, 96, $97,203,220$ bird(s) 59, 96, 151, 152, 153-4, 157, 194-5, 196, 207, 212-13, 214-15, 216, 219,

220, 222, 224, 225n.10

cat(s) 22, 36, 43, 82, 96, 121, 179

$\operatorname{dog}(s)$ 13, 22, 24, 29, 30, 39-41, 54, 76, 102, 139, 141, 171-2, 179, 183, 211, 213-14, 218, 227, 228, 229, 234-6, $237,242 \mathrm{n} \cdot 3 \mathrm{O}$

domestic 2, 34, 38-9, 41, 43, 91, 156, 223, 234-5

fish 214, 203, 220

fox(es) 22, 38, 43, 109, 193, 196, 203, 214

humans as $1,4,11,53-5,61,63,101-8$, $116,147,153,166,168,169-70,173$, 176 n.20, 212, 231, 234, 237

see also becoming-animal

in literature 22, 163

inner see 'beast within'

pig(s) $25,26,54,56,87,96,215-16,217-18$

raising humans $4,8,49,65 n .5,65 n .8$ see also wolf-children

reindeer 227,237

relations with humans 2, 5, 203, 207, 214, 223-4, 237

rights 171,173

seal(s) 213-14, 242n.18

see also selkie

sheep 2, 22, 38-9, 41-3, 42, 46n.20, 87, 235

see also pastoral; pastoralism

stoat 193-4, 196, 197

wild $10,24,34,36,38,43,101-2,103-4$, $105,200,235$ 
animal-human boundary 6, 8, 10, 11, $12-13,71,77,82,83,95,113,114$, $131,147,157,158-9,169-70,172$, $173,175,197,208,214,229,237$, 242n.30

see also human-animal

animality $2,5,6,10-11,12,14,43,48,55$, $71,131,132,138,147,149,150,153$, $157,158,159,160$ n.14, 163, 165, $166,167,169,176$ n.20, 218, 234

anthropocentrism 174-5

anthropology 8, 16n.18, 45-6n.2, 56, 64-5n.5, 204-5

anthropomorphism 25, 165, 208, 241 anti-Semitism 8, 21, 25-6

see also Holocaust, the; Nazis; 'race'

Arbuthnot, John 58

It Cannot Rain But it Pours (1726) 15n.13, 66n.32

Armstrong, Kelly, Bitten (2003) 7, 17n.25 Aroles, Serge 29

art 3, 14, 73, 93, 152, 153, 154, 158, 204, 208, $224,229,234-5$

painting $13,93,104,170,234-5$

photography $193-5,194,195,227,228$, 229-30, 235-41, 239

sculpture and installations 13, 170, 193, 199-203, 200, 201, 204, 205-8, 206

video 193, 194-5, 198-9, 205, 236

Atwood, Margaret 21

Auerbach, Nina 75, 130

D’Aulnoy, Marie-Catherine Le Jumel de Barneville, Baroness see fairy tales autonomy see agency

Baring-Gould, Sabine, The Book of Were-Wolves (1865) 105, 109, 110, 111, 112, 131, 225n.10

Barnes, Djuna, Nightwood (1936) 229

bat(s) see animals

Bear-boy of Lithuania see wild children bear(s) see animals

'beast within' 10-11, 12, 14, 16n.19, 79-8o, 82,128 n.19, 164-6, 168, 175, 211, 218, 221, 231

beauty 72, 96, 98, 129, 138, 236, 237 'Beauty and the Beast' see fairy tales becoming-animal 13, 153, 193-7, 198, 207, 208n.2, 211, 212

see also Deleuze, Gilles; transformation

Being Human (2008-13 TV series) 82, 130, 185

Berger, John 173, 175, 197

Bergson, Henri 197

Berry, Liz 13, 211-18, 221, 222, 223-4

'Bird' 212-13

Black Country (2014) 212

'Carmella' 214-15

'Dog' 213, 218

'Echo' 214, 222

'The First Path' 214

'My Mother's Wedding Shoes' 216

'The Patron Saint of Schoolgirls' 215

'The Red Shoes' 216

'The Sea of Talk' 214

'The Silver Birch' 215

'Sow' 215-16, 217-18, 221, 223-4

'Stone' 213-14

'When I Was a Boy' 215

'Woodkeeper' 214

Bettelheim, Bruno 128n28, 163, 164-5

Beuys, Joseph 199, 209n.25

Bible, The 22, 37, 41, 93, 148, 149, 211 see also Christianity; religion

Big Bad Wolf see Wolf, Big Bad

Bilibin, Ivan 91, 97, 98

binary oppositions 117, 120, 126, 163, 217, 224, 228, 229, 231, 237

birds see animals

'Bisclavret' see Marie de France

blood 6, 14n.8, 74, 109, 111, 116, 159, 167, $183,188,232,234,237$

'Bluebeard' see fairy tales

body, the $49,53,60,63,80,81-2,94,131$, $138,148,157,159$ n.4, 164, 165, 166, 172, 196, 200, 201, 208, 212, 213, $214,215,222-3,224,229,230,231$, 234

female $138,148,212,215,216,217,218$, $231,232,234$

representation of 148, 207-8, 229, 230

transformation of 8o-2, 83n.6, 94, 138, $165,166,212,213,215,222,230,234$ see also flesh; skin 
body horror see horror, body

Boy Who Cried Werewolf, The (1973 film) 80

Bram Stoker's Dracula (1992 film) 83n.6 see also Stoker, Bram

Brontë, Charlotte, Jane Eyre (1847) 107

Browning, Robert, The Ring and the Book (1868) 109

Buffon, Georges-Louis Leclers, Comte de 36,50

see also species

Buffy the Vampire Slayer (1997-2003 TV series) 9, 70, 71, 176n.1

Burgess, Melvin, The Cry of the Wolf (1990) 24

Byron, George, Lord 230

Campbell, Gilbert, 'The White Wolf of Kostopchin' (1889) 231

cannibalism 88, 95, 109, 232

Cantor, Mircea 205

capitalism 12, 148, 150, 152, 154, 158, 159, 16on.17

see also commodification; Marx, Karl

Carter, Angela 4, 12, 13, 147-62, 165, 185, 188,189 . 28

'The Bloody Chamber' 152-3

The Bloody Chamber and Other Stories (1979) 12, 147, 150-9

'The Company of Wolves' 154-6, 165

'The Erl-King' 151-2, 153-4

'The Lady of the House of Love' 153-4

'Peter and the Wolf' (1985) 15n.14, 148-50

The Sadeian Woman (1979) 148, 152, 153, 16on.14

'The Tiger's Bride' 153

'Wolf-Alice' 15n.14, 148, 156-8

see also The Company of Wolves (1984 film); fairy tales

Chaney, Jr, Lon see The Wolf Man (1941 film)

Charnas, Suzy McKee, 'Boobs' (1999) 165

child-rearing 62, 113-14, 116, 117, 120, 121, 123

see also maternity; wolf-children children's literature 24, 69n.63, 163 see also education; fables; fairy tales;

Young Adult fiction

Christianity 2, 41, 48, 56, 95, 136, 148

city, the 11, 23, 30, 48, 90, 110, 132, 140, 150 see also country, the

civilisation $2,3,4,15 \mathrm{n} .13,31,49,56,76,77$, $79,80,87,90,91,131,132,134,137$, 150, 163, 170, 171, 178, 188, 213, 214, 229, 230, 231, 237, 238

see also culture; nature; wilderness; wildness

class 11, 77, 119, 129-43, 16on.14, 212

clothes 42, 43, 56-7, 108, 151, 153, 213, 227-42

see also fashion; fur; skin; transvestism

Coates, Marcus 13, 193-210

Coleridge, Samuel Taylor 61, 67n.44, 232

colonialism 124, 212, 241

see also postcolonialism

commodification; commodities 12, 148, $149,150,151,152-4,155,156,157,159$, 16on.20, 232

see also alienation; capitalism; Marx, Karl; reification

The Company of Wolves (1984 film) 5, 80, 162n.46, 230

see also Carter, Angela

Condillac, Étienne Bonnot de 3, 68n.53, 157

see also language, acquisition of

conservation $37,45,45$ n.2, 47n.27, 206, 207, 220

see also ecology; rewilding

Coudray, Chantal Bourgault du 6-7, 16, $180,216-17,219,221,228$

country, the; countryside 132, 138, 140, 193 see also city, the; forests; pastoralism; wilderness

crime; criminal 1, 9, 11, 37, 38, 42, 42, 74, $87,90,101-12,131,134$

Crozier, Lorna, 'What Comes After' 211

culture $1,3,4,5,6,8,9,12,13,24,34,44$, 45n.2, 62, 96, 147, 149, 150, 153, 155, $156,164,198,203,211,212,219$, 227-8, 229-30, 234, 236, 238, 240, 241

folk 149

popular $80,81,133,163,211$ 
youth 70

see also civilisation, nature, society

The Curse of the Werewolf (1961 film) 80

Dadd, Richard 102, 103-4

Dances with Wolves (1990 film) 238, 240

Darwinism 113, 118, 119, 126, 127, 127n.16

Defoe, Daniel, Mere Nature Delineated (1776) $58,59,67 n .41$

Defonseca, Misha 28-9

degeneration theory 11, 113, 119-20, 124, 126,234

see also Darwinism

Deleuze, Gilles 13, 16n.19, 196-7, 208, 208n.2

see also becoming-animal

Depression, the 8,25

Derrida, Jacques 197

desire 106, 107, 109, 114, 115, 117, 118, 147,

$149,153,155,159,170,216,217,236$, 240, 241

desublimation 148,150

see also sublimation

Dijkstra, Bram 234-5

Dion, Mark 206, 206

disease 104, 129, 132, 167, 169, 173, 183

Disney, Walt 8, 25-6, 27, 73, 163, 212 see also fairy tales

Doctor Who (TV series) 12-13, 178-89

$\operatorname{dog}(\mathrm{s})$ see animals

domestication see animals, domestic

domestic sphere; domesticity 91, 123, 162n.42, 168, 169, 172, 216, 234-5

Doré, Gustave, frontispiece 40

Dracula (1931 film) 73-4, 75-6 see also see Stoker, Bram

Dracula, Count see Stoker, Bram

Dr. Jekyll and Mr. Hyde (1920 film) 74 see also Stephenson, Robert Louis

Dr. Jekyll and Mr. Hyde (1931 film) 70, 73, 79

see also Stephenson, Robert Louis

dualism 148, 212, 217, 241

ecofeminism 212, 217, 223

see also ecology; feminism ecology; environmentalism 5, 7, 9, 13, 34, $35,36,44,181,183,187,205,211,219$, 220, 221, 242n.31

Edgeworth, Maria and Richard 3, 59 see also Peter the Wild Boy

education 11, 56, 58-9, 114, 120-4, 125, 126 see also child-rearing; language, acquisition of

L'Enfant Sauvage (The Wild Child) (1970 film) $68 \mathrm{n} .53$

see also Itard, Jean-Marc-Gaspard; wild children

Engels, Friedrich 150 see also Marx, Karl

Enlightenment, the 1, 2-4, 5, 6, 7, 12, 14, 16n.20, 55-6, 149-50, 157, 180, 228

see also modernity

Enninful, Edward 238-9, 239, 240

environmentalism see ecology

eroticism $6,147,157,162$ n.38, 185, 188, 235

see also desire; sexuality

essentialism 7, 148, 153, 217, 224

evolution see Darwinism

Expressionism 74

fables $8,22-3,39,41,65$ n.10, 189n.28 see also Aesop; fairy tales; genre

fairy tales; folktales 10, 11, 12, 22, 27, 32, 32n.7, 49, 65n.10, 87-10o, 99n.5, 128n.25, 131, 204, 211, 214, 238

Afanas'ev, Alexander Nikolayevich 88, 90, 94

Andersen, Hans Christian 115

D’Aulnoy, Marie-Catherine Le Jumel de Barneville, Baroness 22

'Beauty and the Beast' 22, 154, 161n.33, 163

'Bluebeard' (Perrault) 152, 154, 156

Grimms brothers 22, 65n.10, 88, 89, 128n.25

'Little Redcap' (Grimm brothers) 65n.10, 89,90

'Little Red Riding Hood' (Perrault) frontispiece 2, 5, 8, 12, 14n.6, 22, 65n.10, 94, 150, 151, 155, 164-5, $167-9,172,186,228-9,238$ 
Perrault, Charles 2, 22, 65n.10, 88, 94, 99n.1, 128n.25, 161-2n.36, 164

Russian 10, 87-100

'Sleeping Beauty' 154

see also fables; folklore; genre; myth

fantasy; the fantastic 4, 74, 90, 114, 115, 116, $117-19,124-7,139,217,219,220,221$, 229, 231, 239, 240

see also genre; Gothic

fashion $13-14,227-42$

see also clothes

femininity; feminine, the $6,95,115,123$, 128n.27, 154, 158, 165, 216, 229, 232, 234

see also gender; masculinity; women feminism 6, 7, 12, 153, 154, 16on.9, 161n.26, $165,212,217,218,224$

see also ecofeminism; gender; patriarchy; sexuality; women

Fenrir see myth, Norse

feral children see wild children

fiction 1, 2, 4-7, 8, 10-11, 12, 13, 14, 21, 24, $28,49,50,55,56,62,64,69$ n.63, 79, 105, 107, 109, 124, 130, 147, 166, 170, 172, 204, 211, 231, 234

see also genre; lies; realism; storytelling

flesh 7, 12, 36, 109, 147-62, 219, 234 see also meat; skin

folklore 16n.24, 64-5n.4, 71, 88, 95 werewolves in $2,15 \mathrm{n} .17,16 \mathrm{n} .18,71,11 \mathrm{O}$, 131,183

wolves in $39,87-100$

folktales see fairy tales; folktales

forests $3,10,38,49,56,59,60,62,87-91$, 92, 93, 95-6, 97, 98, 99, 105-6, 110, $111,154,155,167,186-7,188,195$

fox(es) see animals

Frankenstein see Shelley, Mary

Frankenstein (1931 film) 73, 74, 77, 80, 188n.9

Freud, Sigmund 31, 149

Wolf-Man, the 16n.19, 27, 229

see also psychoanalysis

Frozen (2010 film) 73

Frozen (2013 film) 73

fur 13, 83n.6, 89, 91, 94, 108, 141, 153, 156, $178,182,206,215,227-42$

see also hunting; skin furiosus 102, 103, 107, 108, 110 see also mental illness

Galanin, Nicholas 205

Gelder, Ken 179

gender $7,13,14,113,115,116,120-1,122$, $123-4,125,128 \mathrm{n} .27,131,148,165$, $166,168,212,215,216,218,229$, 234

see also femininity; masculinity; sexuality; women

genre $4,5-6,10,11-12,14,15$ n.13, 70, 71, 73, $74,80-1,83,115,124,125-6$

Gilgamesh, Epic of 8, 23

Ginger Snaps (2000 film) 82, 185

Godwin, William 11, 122-3, 126

Gothic 1, 5, 6, 9, 12, 13, 14, 15n.17, 16n.20, $70,74-5,76,147,158,179,180,181$, $211,232,234$

genre $5,6,12,70,130$

horror 4, 5 see also horror

language 105

literature $4,5,6,12,16 \mathrm{n} .20,73,74-5,110$, $163,211,232,234$

mode $1,5,6,158,179,180,181$

Southern 140

see also Harris, Charlaine; True Blood (2008-14 TV series)

see also eco-Gothic; fantasy; genre; horror

Grey Wolf 10, 88, 89, 91, 92, 93-5, 96, 99

Grimm brothers see fairy tales

Guattari, Pierre-Félix see Deleuze, Gilles

gypsies 133,135

\section{Harraway, Donna 131}

Harris, Charlaine, Sookie Stackhouse novels 139 see also True Blood (2008-14 TV series)

Hauser, Kaspar see wild children

heteronormativity 223

see also heterosexuality; homosexuality; sexuality

heterosexuality 138, 212

see also heteronormativity; homosexuality; sexuality

Hobbes, Thomas 131 
Holocaust, the $8,21,26-7,28-9$

fake accounts of $28-9$

see also anti-Semitism; Nazis

homosexuality; lesbianism 129, 185, 188 see also heteronormativity; heterosexuality; sexuality

horror 4, 5, 6, 70, 71, 79, 83, 162n.46, 182, 211,232

body $74,80,180,181$

film 4, 9, 70, 71, 73-5, 76, 80-1, 130, $133,136,162$ n.46, 171, 172, 180

stories 4,32

Housman, Clemence, 'The Were-wolf' (1890) 231, 232-4, 241

Housman. Laurence 233, 233, 242n.2O howl(ing) see wolf/wolves, howl

The Howling (1981 film) 80, 81

Hughes, Ted

'The Howling of Wolves' 64n.2

'Life After Death' 9, 48-9, 64

Lupercal (1960) 64n.2

Wolfwatching (1989) 64n.2

human-animal 12, 60, 113, 158, 163, 175, 197, $212,229,235,242 n \cdot 30$

relations 5, 96, 197, 199, 203, 208

see also animal-human boundary; binary oppositions; transformation

humanism 7, 12, 147, 150, 153, 159, 161n.26, $161 n .30$

see also anthropocentrism

hunters; hunting $8,10,24,30,34,38,39$, $45,51,50,87,90,111,121,171,188$, $205,223,227,232,235$

see also fur; skin; wolves, as hunters 10, $34,35,36,38,89,90$

hybridity $5,12,147,156,158,163,166,170$, 179, 180, 181, 182, 183, 184, 187, 211, 224

identity $5,7,10,13,16,163,164,166,167$,

$170,171,173-5,181,197,216-18,223$,

224, 228-9, 233-4, 237

see also agency; subjectivity

illustration 42, 91, 124, 232-4

imitation 26, 57, 58, 59, 196, 208

of animals 57, 58, 60, 193, 196 see also becoming-animal immigration 9, 131

see also migration; postcolonialism; 'race'

indigenous peoples 13-14, 137, 142, 211, 220-1, 229, 231, 236-41

insanity see mental illness

instinct 4, 7, 25, 30, 38, 54, 147, 149, 153-4, 157 , 16on.8, 16on.9, 164, 174, 180, 222, 223

intertextuality 5, 12, 70, 81, 157, 179, 225n.14, 240

Itard, Jean-Marc-Gaspard 15n.9, 55, 61, $68 \mathrm{n} .53$

see also L'Enfant Sauvage (The Wild Child) (1969 film); wild children

I Was a Teenage Werewolf (1957 film) 80

Kipling, Rudyard 4, 24, 31, 51

Mowgli 24, 30, 51, 52, 55

see also colonialism; wolf-children

Kristeva, Julia 16n.19, 149, 16on.8, 16on.14, 170

see also abjection; language

Kubrick, Stanley 8, 26-8

Lacan, Jacques 157

see also language

Lamb, Mary 102-3, 104, 109

language $3,5,7,8,13,62,64,106,148$, $149,150,153-4,156,157,158,163$, $166,170-3,174-5,183,216,218$, 238

acquisition of 31, 48, 56, 59-60, 61, 64, 156 see also education

non-verbal 59-6o, 62-3, 148, 156, 157, $170,171-2$

origins of 2, 3, 8, 157

Lauren, Ralph 13, 227, 228, 229, 234-8, 240-1

Laurence, H., 'Norman of the Strong Arm' (1827) 195

Leblanc, Marie-Angélique see wild children

Lévi-Strauss, Claude 228

Lewis, C.S. 24

lies $8,21,22,26,27,28-9,30,32$

see also wolves, as deceivers

liminality $88,95,131-3,164,169,175,229$,

237 
'Little Red Riding Hood' see fairy tales

'Little Redcap' see fairy tales

Locke, John 9, 11, 48, 56, 59, 68n.53, 120,

$$
121,122,126
$$

see also education

Lon Chaney, Jr see The Wolf Man (1941 film)

London, Jack, White Fang (1906) 24

loup-garou $133,167,178$

see also werewolf

lunacy see mental illness

lupicide 50, 204

see also wolves, extinction of

lupophilia 204, 205

lupophobia 2, 8, 34, 43, 50, 72-3, 175, 204, 205

lycanthropy 5, 6, 12, 13, 105, 106, 129, 131, $132,151,156,165,166,167,168,171$, $174,175,178,179,180,182-6$ passim, 219, 230

as psychological disorder 10, 102, 105, 106, 111, 131, 132, 166

see also werewolf

Lycaon 4, 184

see also Ovid

McCarthy, Cormac, The Crossing (1994) 24

MacDonald, George

'The Gray Wolf' (1871) 231

'The History of Photogen and Nycteris' (1879) 11, 113-28

see also fairy tales

McNaghten, Daniel 101, 103, 104, 107, 108, 109

McNaghten Rules 104-5, 108-9, 110

madness see mental illness

magic $11,88,89,94,108,115,116,117-19$ see also fairy tales; supernatural

Marcuse, Herbert 16n.19, 149, 159, 159n.5, 16on.9, 161n.22, 162n.38, 162n.47 see also Marx, Karl

Marryat, Captain Frederick, 'The White Wolf of the Hartz Mountains' (1839) 231-2

Marvin, Garry 8, 50, 72, 73, 204-5, 230

Marx, Karl 150, 152, 157, 159 see also capitalism; commodification; Engels, Friedrich masculinity $95,156,158,165,216,217$, 230-1, 232

see also femininity; gender; sex; sexuality

materialism 12, 153, 159, 161n.26, 161n.33

maternity; motherhood 4, 30, 48, 51, 53, 63, 97, 113-14, 116, 123, 138

see also child-rearing; women

meat 12, 54, 87, 96, 106, 109, 147, 148, 151, $152-3,154,155-6,158,162$ n.39, 165 , 224, 237, 238

see also animals; body, the; flesh; skin melancholy 9, 62, 64, 71, 72, 131, 182 menstruation 157,165

mental illness 11, 69n.61, 101-12

and animality 104, 110-11

and the law

see also moral insanity

Menzies, Sutherland, 'Hughes, The Wer-Wolf’ (1838) 105, 106

metafiction 13, 182

metamorphosis see transformation

metaphor see werewolf, as metaphor; wolf, as metaphor

Meyer, Stephenie, Twilight series (books) 11, 129-30, 133, 136-9, 166, 173-4, $176 \mathrm{n} .2 \mathrm{O}$

see also Twilight Saga (films)

Midgley, Mary 173

Milton, John 127.8

mimesis see imitation

minimalism 13, 199, 202-3, 208, 210n.62

see also art

M'Naughton see McNaghten

modernity 2, 6, 8, 11, 14, 15n.13, 24, 30, 148, 149, 180,181

see also Enlightenment, the

Monboddo, James Burnett, Lord 3, 157

monsters; monstrosity 2, 5, 6, 10, 12, 13, 23, $34,43,53,71,76-80$ passim, 82,83 , 97, 120, 129, 130-3 passim, 136, 139, $141,142,147,151,154,158,163,166$, $168,169,171,172-3,178-82$ passim, $185,187,228$

Montesquieu, Charles-Louis de Secondat, Baron de La Brède et de 58

moon see wolves, and the moon; werewolves, lunar influence on 
Moore, Kim 13, 211-12, 218-24

'And the Soul' 221-2

The Art of Falling (2015) 218, 219

'Body, Remember' 223

'How I Abandoned My Body to His Keeping' 222, 223

'How the Stones Fell' 218

'How Wolves Change Rivers' 220-1 If We Could Speak Like Wolves (2012) 219

'If We Could Speak Like Wolves' 219

'My People' 222

'Picnic on Stickle Pike' 218-19

'That Summer' 218

'Translation' 222

'When I Was a Thing with Feathers' 222 moral insanity 11, 108-9, 110

see also mental illness

Morris, Robert 199, 201, 202-3, 207, 208

Moss, Kate 235

Mowgli see Kipling, Rudyard

music 9-10, 57, 61, 62, 70-1, 72, 73, 75, 78, $79,81-2,122,157,240$

myth 4, 8, 23, 43, 48, 49, 55, 64, 64-5n.4, 141, 170, 178-9, 181-2, 184, 185, $187-8,204,214,217,225 n .10,227$, 228,240

Germanic 24

Greek 4, 8, 23

Hebrew 149

Native American 23-4

Norse 8, 23, 225n.10

Roman 4, 8, 23, 49

see also folklore; religion

Nagel, Thomas 195-6

Native Americans 11, 23-4, 137-8, 176n.20, $231,238,240$

see also indigenous peoples

nature 1-9 passim, 12, 13-14, 34, 35-6, 43, $44,45,46$ n. $4,48,56,58,59-6$ o, $62-4,89,95,118,125,132,138$, 147-51 passim, 153, 154-7, 158, 162n.38, 163, 164, 167, 169, 171, 178, $187,197,203-4,212,223,227-8,229$, 230, 234, 239, 240, 241

human 1, 3, 10, 44, 58, 66n.28, 68n.49, $95,103,108,148$ state of $9,48,56,59,60,63,64$ of wolf see wolf, nature of

see also culture; wilderness; wildness

nature-culture 1, 4, 12, 56, 212, 229

see also binary oppositions

Nazis 8, 25-8

see also anti-Semitism; Holocaust, the

Nelson, Jimmy 13, 227, 236-8, 240

noble savage 31,63

see also indigenous peoples; nature;

Rousseau, Jean-Jacques; wild man; wildness

non-human 45, 91, 120, 159, 163, 170, 171, $196,197,199,207,208,212,214,216$, 223, 224

see also animal(s); nature

Nosferatu (1922 film) 74

Original Sin 48, 56, 6o, 66n.28

see also Christianity; noble savage

Otherness; Other, the 5, 8, 13, 15n.13, 6o, $76,83,108,130-3$ passim, 136, 139, $142,150,154,163,164,166-73$ passim, 175, 180, 196, 197, 217, 234

Ovid 4, 65n.7, 184, 223

painting see art

paranormal romance 1, 4, 5-6, 7, 12, 147, $151,154,158,159$ n.1, 162n.46, 164 see also desire; genre; romantic fiction; Young Adult fiction

pastoral (genre) 1-5 passim, 7, 8, 14, 15n.13, 149

see also genre

pastoralism; pastoral societies $2,3,5,8,10$, $34,39,41,87,149$

patriarchy 10, 12, 13, 132, 149, 151, 152, 159, 16on.17, 219, 223

see also feminism; women

performance $13,75,77,133,134,182,183$, 193, 196-9, 200, 207-8, 214, 215, $218,237,238,240$

principle 162 n. 47

Perrault, Charles see fairy tales

Peter the Wild Boy (Peter of Hanover) see wild children

Petronius, Satyricon 230

The Phantom of the Opera (1925 film) 70, 74 photography see art 
$\operatorname{pig}(\mathrm{s})$ see animal(s)

Piggott, Marcus 238

Plath, Sylvia 48

poetry $9,14,48,56,59-60,61-4,172$, 211-26

postcolonialism 220-1

see also colonialism

postmodernism 7, 15n.20, 81, 217

postmodernity 184, 211

poststructuralism 217

Priest, Hannah 16, 230, 232

Propp, Vladimir 97

see also fairy tales; folk tales

puberty see adolescence

'race'; racism 119, 124, 125, 131, 141, 176n.20, 212,240

see also anti-Semitism; postcolonialism rationalism $16 \mathrm{n} .20,18 \mathrm{o}$

rationality $10,16 \mathrm{n} .2 \mathrm{O}, 56,58,63,101-2,109$, 110, 116, 119, 126, 149, 158, 164, 18o, 187

realism 21, 25, 119, 158, 162n.44, 206, 239 magical 89

reification 12, 147, 151, 154, 16on.17, 161n.26, 162n.39, 229

see also alienation; commodification

reindeer see animal(s)

religion 93, 94, 95, 115, 216

see also Christianity; myth

repression 148, 149, 150, 153, 159, 185, 229

see also sublimation

rewilding see wolves, rewilding

Reynolds, G.M.W., Wagner the Wehr-wolf 4, 11, 106-8

Rice, Anne, The Wolf Gift (2010) 7

Rihanna 238-40, 239

Rilke, Rainer Maria 1, 170, 171, 172

Ritchie, Leitch, 'The Man-Wolf' (1830) 105

ritual 198-9, 227, 230, 239

Robinson, Mary, 'The Savage of Aveyron'

$$
\text { 9, 61-4 }
$$

romance (genre) 1, 4

see also genre; paranormal romance

romance (love) 6, 12, 27, 91, 93, 138, 139, $141,151,154,158,163,167,238$

romantic fiction 5, 6, 12, 158, 162n.46, 164 see also genre; paranormal romance
Romanticism 1, 7, 9, 48, 60, 61-4

Romulus and Remus see wolf-children

Rousseau, Jean-Jacques 3, 9, 11, 31, 48, 56, $58,59,63,113,120-2,123,126,157$

Rowling, J.K., Harry Potter (novels and films) 134

Sade, Donatien Alphonse François, Marquis de 152, 159

science $4,9,11,16 \mathrm{n} .20,35-6,37,45,76$, $77,113-28,129,168,171,179,18$ o, 187

see also technology

science fiction 180, 181, 184

see also fantasy; genre

Sconduto, Leslie 16, 181

sculpture see art

seal(s) see animal(s)

Sedgwick, Marcus, The Dark Horse (2002)

$$
8,24,30,69 n .63
$$

self-consciousness 158, 211, 239, 240

see also subjectivity

selkie $96,242 n .18$

see also seal(s); shapeshifters

sex 27, 93, 94, 122, 141, 151, 152, 154, 158, 159, $169,185,212,214,215,217,219,228$, 229, 238

sexuality $4,5,12,13,116,132,140,147,148$, $162 \mathrm{n} .47,163,164-5,167-8,169,212$, $215,217,218,234$

Shakespeare, William

The Tempest 179

The Winter's Tale 49, 62

shamanism 13, 95, 193, 197-9, 198, 239, 240

shapeshifters $4-5,6,10,12,17 n .25,72$, 83n.6, 88, 89, 96-7, 131, 137, 139, 164, $166,178,181,188,204,211-12,213$, $217,221,222,231,242 \mathrm{n} .18$

see also loup-garou; selkie; werecat; werewolf

sheep see animal(s)

Shelley, Mary, Frankenstein (1818) 113

shepherds 2, 22, 23, 39, 40, 41, 42, 42, 45-6n.2, 46n.20, 49

see also animals, sheep; pastoral; pastoralism

she-wolf see wolf; wolves She-wolf of London (1946 film) 132 
The Shining (1989 film) see Kubrick, Stanley

silence $73,75,213$

see also music; sound (in film)

Silver Bullet (1985 film) 80

silver bullets 172

Sirius Black (see Rowling, J.K.)

skin $1,42,45,46 \mathrm{n} .20,81,82,96-7,106,107$, $108,124,151,152-3,156,158,166$, 178, 198, 199, 205, 213, 214, 225n.10, 227, 229-33 passim, 235, 238

see also flesh; fur; hunting; meat 'Sleeping Beauty' see fairy tales snow 73, 168, 227, 234, 236-7 socialism 12,161 n.26 see also capitalism; Marx, Karl sociality $2,3,14,150$

sociobiology 7

song see wolf, song; music

Sorrenti, Mario 235

sound $9,10,48,55,61,70-84,157,180$ effects $71,73,75,78,79,82$ see also film; music; silence; transformation, sound of special effects (in film) 80, 81 see also sound, effects

species $1,6,23,37,38,57,106,138,139,163$, 173, 181, 197, 200, 203, 207, 212, 217, 220, 224, 229, 231, 234, 241

speech see language

Stevenson, Robert Louis, Dr Jekyll and Mr Hyde (1886) 132, 179

see also Dr. Jekyll and Mr. Hyde (1920 film); Dr. Jekyll and Mr. Hyde (1931 film)

stoat see animals

Stoker, Bram, Dracula (1897) 10, 16-17n.24, 71-2, 83n.6, 132, 133, 135-6, 240

Stone, Lara 235

storytelling 4, 5, 8, 9, 21, 23, 25, 28, 32, 48, $51,55,62,64,187,204,224,227$ see also folk tales

Stubbe, Peter (also Stube, Stump, Stumpf) $1,46-7 n .23$

subjectivity $7,147,149,153,164,170-1,174$, 175

see also agency; identity; self-consciousness sublimation $148,153,155,159,159$ n. 5 see also desublimation; psychoanalysis supernatural 2, 6, 53, 88, 89, 99, 108, 114, $133,136,137,139,141,158,171,180$, 187,234

see also magic

Suvin, Darko 124

Sweeney Todd 109, 110

Swift, Jonathan, The Most Wonderful Wonder that ever appeared to the Wonder of the British Nation (1726) 15n.13, 66n.32

symbolic order 157,228 see also Kristeva, Julia; Lacan, Jacques; language

sympathy $10,50,62,64,82,126$

tabula rasa see Locke, John

technology 73, 101, 180, 181, 182, 183 see also science

teenagers 114, 138, 163-77, 185, 216 see also adolescence; Young Adult fiction

Teen Wolf (1985 film) 80, 133, 165

Teen Wolf (2011- TV series) 165, 184

Teffi, Nadezhda 98-9

Thomson, Richard, 'The Wehr-Wolf' (1828) 105-6

The Three Little Pigs (1933 film) 8, 25-7

Tolkien, J.R.R. 24

transformation $14,43,88,89,103,148,150$, $153,155,159,164,180,181,182,183$, $187,188,213,223,224,230,239,240$, 241

of genres 2, 12, 55, 56, 147, 157, 16on.17, 162n.46, 183

human/animal 12, 83n.6, 96, 150, 152-3, $157,178,179,188,211-24,225$ n.10, 229

human/(were)wolf 13, 43-4, 78, 79-82, 106-8, 109, 110, 113, 117, 119-20, 129, $130,133,134,135,138,156,157,158$, 164-7 passim, 169, 170, 171-5, 219, $221,230,235,241$

sound of $10,71,77,81-2$

transvestism 13, 228-9, 234, 241 see also clothes; skin 
True Blood (2008-14 TV series) 11, 130, $133,139-41$

see also Harris, Charlaine

Twilight (2008 film) 11, 138

Twilight Saga (films) 11, 137, 231

Twilight series (books) see Meyer, Stephenie

Underworld (film series) 133, 184

The Undying Monster (1942 film) 132

Universal Studios 71, 73, 74, 76-7, 80, 81, $129,133,134,179,188 \mathrm{n} .9,230$

see also horror, film

urban life see city, the

utopianism 1, 4, 7, 14, 63, 149, 153, 157, 158, 159,16 on.13

vampires 2, 5, 6, 10, 11, 16-17n.24, 71, 74, 111, 130-4 passim, 136, 138, 139-40, 141-2, 147, 166, 178, 180, 184-5, 230, 231, 232

Vampires Suck (2010 film) 138

Van Helsing (2004 film) 133

Vasnetsov, Victor 91, 92

Victor of Aveyron see wild children video see art, video

Vincent, Rachel, Stray (2009) 17n.25

Vloet, Sanne 227, 228, 237, 240

Walsh, Jill Paton, Knowledge of Angels (1994) 4, 30-1

werecat $17 n .25,188$

weres see shapeshifters

The Werewolf (1913 film) 184

Werewolf of London (1935 film) 4, 10, 71, $76,77-80,129,133,134,179,185$

werewolves

in $\operatorname{art} 13$

Classical 4, 9, 34, 230

contemporary 2, 4, 5-7, 44, 211, 219

female $5,6-7,13-14,96,113-28,132$, $140-1,147,159$ n.1, 165, 166, 167-8, 171-4, 175, 219, 222, 227, 229-34, 240, 241

in film 4, 9-10, 11, 70-84, 129-43, 179, 188 n.9, 230

in folklore 10, 12, 71, 119, 131, 164-5, 179, 211, 227 and Gothic 5, 14, 147, 211

involuntary 107

in literature 12,13

lunar influence on $72,77,81,107,133$

and madness 10-11, 102-12

medieval 9, 34, 43, 110, 166, 211, 230

as metaphor 5, 10, 12, 106, 131, 136, 142, $163,167,168-70,175,182$

and nature $1,2,5-8$ passim, 12, 13-14, $111-12,147,164,169,187,227,229$, 230, 234, 237, 240

sympathetic 5-6, 77, 79, 82-3, 130, 147, 159n.1, 163-77, 219

transformation into, see transformation in TV $11,12-13,129-43,178-89$

as victims see werewolves, sympathetic victims of 77,78

Victorian 10, 11, 105-8, 110, 231-4

voluntary 96, 107

and wolves 1, 4, 8, 34, 38, 39, 43-4, $46-7$ n.23, 188, 204, 221

working-class 11, 129-43

see also loup garou; shapeshifters

What We Do in the Shadows (2014 film) 141

Whedon, Joss see Buffy the Vampire Slayer wild children 1, 3, 9, 14, 15n.9, 48-69, 228

Bear-boys of Lithuania 49, 50, 65n.5

Blanc, Marie-Angélique, Le (The Wild Girl of Champagne) 3, 29, 156

feral children $3,8,13,28-9,30,49,55-6$, $63,188,238,239$

Hauser, Kasper 55-6, 228

and language 3, 8, 156, 157, 228, 238

in literature $4,8,9,48-69,148$

Peter the Wild Boy (Peter of Hanover)

$3,4,9,15 n .13,55-9,60,63,64$, $65 n .5,67 n .33,67 n .35,67 n .44$, $69 n .63$

raised by animals $3,4,5,8,56,148,156$ see also wolf-children

Victor of Aveyron 4, 9, 55, 56, 60-2, 63, 64, 57n.44, 68n.53, 69n.61, 69n.63

wilderness 8-11 passim, 13, 14, 30, 35, 36, $44,73,88,130,156,163,168,180$, $187,204,205-6,220,227,229,231$, $234,236-8,240,241$

see also nature 
Wild Girl of Champagne, The (MarieAngélique Le Blanc) see wild children

wild man 15n.13, 57, 179

see also noble savage; wild children

wildness 4, 8, 9, 29-32, 41, 44, 57, 59, 71, $96,97,102,150,153,154,156,222$, $231,238,240$

witches $10,11,24,87,89,90,95,96,97,99$, $113-28$

Baba Yaga 88, 89, 91, 94-9

Wolf (1994 film) 133

wolf; wolves

'at the door' 27

behaviour 9, 36

'boy who cried' 21

children raised by

see wolf-children

communication $38,156,170,171-2$, 219, 222

as deceiver 8, 21-33, 39, 41-2, 43, 169

extinction of 1, 24, 50, 111, 203-4, 226n.32

fear of see lupophobia

as helper 3, 4, 10, 23, 28, 49, 64, 87-100, 187

howling 9, 10, 48-9, 70-1, 73, 76, 77, 78, $82,154,156,157,221,224$

inner see 'beast within'

'in sheep's clothing' 21, 111

as metaphor 10, 24, 27, 87, 165, 169, 219

and the moon 72

nature of 9, 21, 23, 24, 25, 34, 35-6, 38, $39,41-2,44,50,89,117,167,169$, 232

as noble $8,24,27,53$

as outlaw 105

pack $2,6,7,17$ n.25, 24, 29, 30, 31, 35, $36,38,51,71,73,130,139,140$, $148,168,175,186,196,206,234$, 241

see also wolf, as social

as predator $2,3,10,23,24,25,34,36-7$, $37,43,87,103,147,154,172$ rewilding 2, 9, 44-5, 130, 142, 204-5, 220, 224

as sexual predator $2,27,93,155,165,168$, 169

she-wolf 49, 58, 67n.41, 132, 222, 232

skin 1, 106, 107, 108, 205, 225n.10, 227-42

see also fur; skin

as social $2,6,35,37-8$

teeth $36,37,72,91,93,167,181,182,223$

whistle 2

see also animal(s)

Wolf, Big Bad 2, 25, 27, 39, 65n.10, 141, 165, 186

wolf-children $8,9,10,12,29,30,48-69$

Amala and Kamala 4, 15n.14, 29, 48, $53-5,54,60,61,64,69 n .63$

Mowgli see Kipling, Rudyard

Romulus and Remus 3, 23, 28, 49, 51, 51, $64 \mathrm{n} .2,67 \mathrm{n} .41$

see also wild children

Wolfen (1981 film) 80

The Wolfman (2010 film) 133

The Wolf Man (1941 film) 4, 10, 11, 76, 77, 79, 8o, 83, 129, 133-6, 185, 188n.9, 230

Wolf-Man, the see Freud, Sigmund wolf-skin see fur; wolf/wolves, skin women $5,6-7,12,13-14,90,96-7,114$, $115-16,140-1,154,165,212,214,219$, 222, 224, 228, 229, 231, 234-5, 241

see also feminism; gender; sex

Wordsworth, William 9, 61, 62, 67n.44, $67-8 n .46,68 n .57$

'The Idiot Boy' (1798) 59-6o, 61

Young Adult fiction 1, 4, 12, 163-77 see also adolescence; children's literature; paranormal romance; teenagers

Zipes, Jack 115, 164

Žižek, Slavoj 18o, 228

zoanthropy 53

see also lycanthropy

Zvorykin, Boris Vasilyevich 91 